\title{
Twisted topological solitons and dislocations in a polymer crystal
}

\author{
Savin, A. V.; Khalack, J. M.; Christiansen, Peter Leth; Zolotaryuk, Alexander
}

Published in:

Physical Review B Condensed Matter

Link to article, DOI:

10.1103/PhysRevB.65.054106

Publication date:

2002

Document Version

Publisher's PDF, also known as Version of record

Link back to DTU Orbit

Citation (APA):

Savin, A. V., Khalack, J. M., Christiansen, P. L., \& Zolotaryuk, A. (2002). Twisted topological solitons and dislocations in a polymer crystal. Physical Review B Condensed Matter, 65(5), 054106.

https://doi.org/10.1103/PhysRevB.65.054106

\section{General rights}

Copyright and moral rights for the publications made accessible in the public portal are retained by the authors and/or other copyright owners and it is a condition of accessing publications that users recognise and abide by the legal requirements associated with these rights.

- Users may download and print one copy of any publication from the public portal for the purpose of private study or research.

- You may not further distribute the material or use it for any profit-making activity or commercial gain

- You may freely distribute the URL identifying the publication in the public portal 


\title{
Twisted topological solitons and dislocations in a polymer crystal
}

\author{
A. V. Savin, ${ }^{1,2}$ J. M. Khalack, ${ }^{1,3}$ P. L. Christiansen, ${ }^{1}$ and A. V. Zolotaryuk ${ }^{1,3}$ \\ ${ }^{1}$ Section for Mathematical Physics, IMM, Technical University of Denmark, DK-2800 Lyngby, Denmark \\ ${ }^{2}$ State Institute of Physics and Technology, 119034 Moscow, The Russian Federation \\ ${ }^{3}$ Bogolyubov Institute for Theoretical Physics, 03143 Kyiv, Ukraine
}

(Received 13 July 2001; published 9 January 2002)

\begin{abstract}
Topological defects and dislocations in strongly anisotropic crystals consisting of parallel molecular chains are investigated. Our study is focused on the defects in crystalline polyethelyne, which are formed by transverse displacements of chain molecules (mutual substitutions and interlacings of adjacent molecular chains in the polymer crystal). It is shown that some of these defects called "twisted topological solitons" can propagate with a stationary profile and velocity. To describe the dynamics of these solitons, a model that accounts for the three components of the molecular displacements is suggested. Linear topological defects-dislocations-that incorporate the bending of molecular chains in the crystal are also studied.
\end{abstract}

DOI: 10.1103/PhysRevB.65.054106

PACS number(s): 63.20.Pw, 61.72.Lk, 63.20.Ry, 63.90.+t

\section{INTRODUCTION}

The well-known one-dimensional (1D) FrenkelKontorova (FK) model, ${ }^{1-3}$ originally introduced for description of dislocation dynamics in 3D crystals,,${ }^{4,5}$ has been used extensively for modeling nonlinear dynamical processes in a variety of condensed matter ${ }^{6,7}$ and biological ${ }^{8}$ systems. As for possible extensions of this model to higher dimensions, so far little work has been done. In this context, investigations of the $2 \mathrm{D}$ scalar $^{9-11}$ and vector ${ }^{12-14}$ lattice models, including different quasi-one-dimensional ladders ${ }^{15-18}$ that appear also to be complicated systems, should be mentioned.

In the theory of dislocations, ${ }^{4,5}$ the 1D FK model describes the simplest physical situation when a part of a crystal is displaced with respect to another part along a sliding plane. Both these parts, which are separated by a sliding plane, are modeled by 1D discrete chains. The lower chain is considered as a perfect 1D periodic substrate lattice, whereas the upper one is assumed to contain a defect: a localized rarefaction (kink) or a localized compression (antikink). However, in realistic crystals, any disturbance in the upper chain obviously has an influence on the lower lattice, so that the periodicity of the substrate potential in general will be broken. Both chains should be considered as equivalent objects, and the influence of any local stress deformation on the substrate potential should be properly considered. To this end, recently ${ }^{19}$ we suggested a model for an anisotropic crystal consisting of coupled chains which are considered identical objects. In this model, any influence of one of the chains on its surrounding chains is taken into account. As in all the 2D lattice models studied previously, ${ }^{10-14,20,21}$ only interatomic forces were included in this anisotropic crystal model. As a consequence, on-site potentials were not considered at all. Instead, all long-range interactions between atoms in adjacent chains have been considered. In this anisotropic model, only topological defects and dislocations formed due to displacements along the anisotropy axis were studied.

This paper aims at studying topological defects and dislocations formed by transverse displacements resulting in a twist of adjacent chains of the crystal. However, in order to describe defects of this type properly, interactions with all neighboring chains must be involved in the model, not only the four nearest-neighbor chains as before. ${ }^{19}$ Therefore, this problem appears to be much more complicated compared with the case of defects formed only by longitudinal displacements. ${ }^{19}$

For the lattice model of a strongly anisotropic crystal, we choose a 3D polymer crystal that consists of interacting parallel molecular chains. The molecules within each chain are coupled among themselves by strong valence bonds. The interchain interactions are realized through considerably more feeble nonvalence bonds. The molecular chains in such a strongly anisotropic crystal cannot be torn. This property leads to the existence of specific topological defects and to specific features of dislocations.

It is commonly used to regard the polymer crystal as a quasi-one-dimensional structure, for which one can restrict oneself to consider only longitudinal deformations of the links in molecular chains. This approach allows us to examine the existence of "longitudinal" topological solitons in the crystal and edge dislocations with a Burgers vector parallel to the direction of molecular chains, ${ }^{19}$ but it does not allow us to consider topological defects and dislocations, the existence of which is due to transverse deformations of the molecular chains.

In the present paper, a more general 3D model that takes into account for all three components of molecule displacements is elaborated. This model allows a consideration of some new kinds of point topological defects (formed because of interlacings of chains or mutual substitutions of chains) along with a wider class of linear topological defects.

\section{MODEL}

We consider a three-dimensional anisotropic crystal consisting of interacting molecular chains as shown in Fig. 1. Each of these chains is directed along the $z$ axis. The distances between the molecules in the perpendicular $x, y$, and $z$ directions are given by parameters $a, b$, and $c$, respectively. The crystal sites are numbered along the $x, y$, and $z$ axes by indices $m, n$, and $p$, respectively. Then the site $(n, m, p)$ of the $3 \mathrm{D}$ lattice has the coordinates 


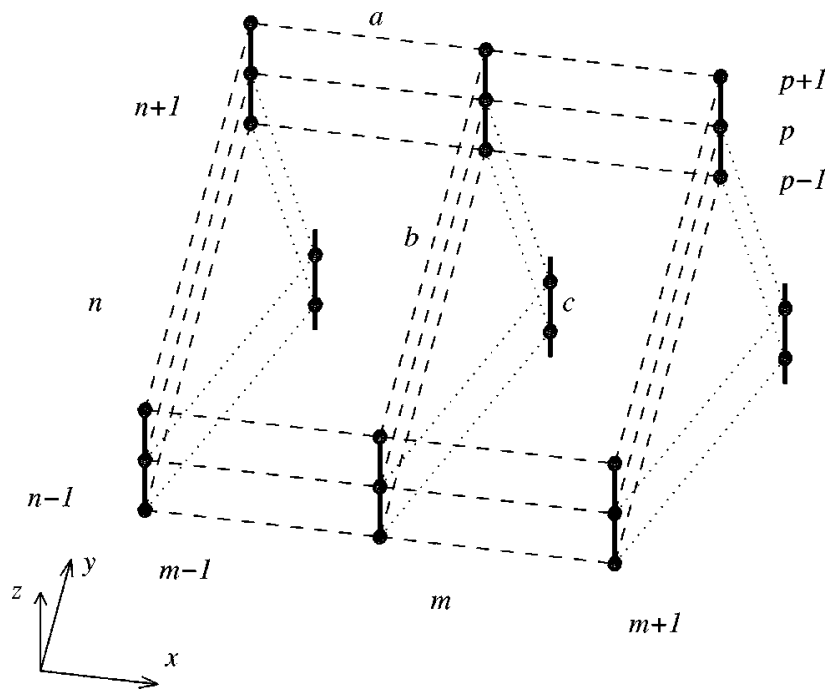

FIG. 1. Schematic representation of a 3D polymer crystal structure. Only intrachain bonds are represented (shown by bold lines).

$$
\begin{gathered}
x_{n m p}=\left(m+\delta_{n}\right) a, \quad y_{m n p}=n b / 2, \\
z_{n m p}=\left(p+\delta_{n}\right) c, \quad \text { where } \delta_{n}=\left[1+(-1)^{n}\right] / 4 .
\end{gathered}
$$

In the $x y$ plane, we have hexagonal close-packed lattices (each chain is equidistant from its six nearest chains). Parameter $a$ specifies the distance between adjacent chains, and $b$ $=a \sqrt{3}$.

Within each chain, the interaction of adjacent molecules is given by the harmonic potential

$$
U(\rho)=\frac{1}{2} K_{\rho}\left(\rho-\rho_{0}\right)^{2},
$$

where $K_{\rho}$ is longitudinal stiffness of a chain, $\rho$ is an instantaneous bond length, and $\rho_{0}$ is an equilibrium bond length in the isolated chain. We describe the energy of bending deformations of each chain in the crystal by the potential

$$
W(\theta)=K_{\theta}(1+\cos \theta),
$$

where $\theta$ is a bending angle of the chain ( $\theta=\pi$ for equilibrium), and $K_{\theta}$ is a chain bending stiffness constant. The interchain interaction of molecules is given by the Morse potential

$$
V(r)=\varepsilon\left\{\exp \left[-\beta\left(r-r_{0}\right)\right]-1\right\}^{2},
$$

where $\varepsilon$ is the binding energy, $\beta$ describes the curvature of the potential minimum, and $r$ is an instantaneous length of intermolecular distance, with $r_{0}$ being its equilibrium.

The values of parameters $\rho_{0}$ and $r_{0}$ are uniquely determined by the lattice periods $a$ and $c$. In equilibrium, the energy per each lattice site reads

$$
E_{0}=\frac{1}{2} K_{\rho}\left(c-\rho_{0}\right)^{2}+\frac{1}{2} \sum_{(m, n, p) \in \mathrm{Z}^{3}} V\left(r_{m n p}\right),
$$

where distance $r_{m n p}$ is given by

$$
r_{m n p}=\sqrt{\left[\left(m+\delta_{n}-1 / 2\right)^{2}+3 n^{2} / 4\right] a^{2}+\left(p+\delta_{n}-1 / 2\right)^{2} c^{2}} .
$$

The set of equations of equilibrium is as follows

$$
\begin{aligned}
\frac{\partial E_{0}}{\partial a} & =\sum_{(m, n, p) \in Z^{3}} V^{\prime}\left(r_{m n p}\right)\left[\left(m+\delta_{n}-1 / 2\right)^{2}+3 n^{2} / 4\right] a / 2 r_{m n p} \\
& =0 \\
\frac{\partial E_{0}}{\partial c}= & K_{\rho}\left(c-\rho_{0}\right) \\
& +\sum_{(m, n, p) \in Z^{3}} V^{\prime}\left(r_{m n p}\right)\left(p+\delta_{n}-1 / 2\right)^{2} c / 2 r_{m n p}=0
\end{aligned}
$$

The values of the parameters $r_{0}$ and $\rho_{0}$ are uniquely determined from nonlinear equations (7) and (8), respectively.

Let $u_{m n p}, v_{m n p}$, and $w_{m n p}$ be the displacements from the equilibrium position for a lattice site $(m, n, p)$. Then the Hamiltonian of the polymer crystal takes the form

$$
H=\frac{1}{2} \sum_{(m, n, p) \in \mathrm{Z}^{3}} M\left(\dot{u}_{m n p}^{2}+\dot{v}_{m n p}^{2}+\dot{w}_{m n p}^{2}\right)+E,
$$

where $M$ is mass, and the potential energy is given by

$$
\begin{aligned}
E= & \sum_{(m, n, p) \in \mathrm{Z}^{3}}\left[U\left(\rho_{m n p}\right)+W\left(\theta_{m n p}\right)\right. \\
& \left.+\frac{1}{2} \sum_{(i, j, k) \in \mathrm{Z}^{3}} V\left(r_{m n p ; i j k}\right)\right] .
\end{aligned}
$$

Here the distances between adjacent molecules within one chain are

$$
\rho_{m n p}=\sqrt{a_{m n p}^{2}+b_{m n p}^{2}+c_{m n p}^{2}},
$$

where

$$
\begin{gathered}
a_{m n p}=u_{m, n, p+1}-u_{m n p}, \\
b_{m n p}=v_{m, n, p+1}-v_{m n p}, \\
c_{m n p}=c+w_{m, n, p+1}-w_{m n p} .
\end{gathered}
$$

The angle $\theta_{m n p}$ is given by

$$
\begin{aligned}
\theta_{m n p}= & \arccos \left[-\left(a_{m n p} a_{m, n, p+1}+b_{m n p} b_{m, n, p+1}\right.\right. \\
& \left.\left.+c_{m n p} c_{m, n, p+1}\right) / \rho_{m n p} \rho_{m, n, p+1}\right] .
\end{aligned}
$$

The distance between the sites $(m, n, p)$ and $(m+i, n+j, p$ $+k)$ can be written as

$$
\begin{aligned}
r_{m n p ; i j k}= & \left\{\left[\left(i+\delta_{n+j}-\delta_{n}\right) a+u_{m+i, n+j, p+k}-u_{m n p}\right]^{2}\right. \\
& +\left[v_{m+i, n+j, p+k}-v_{m n p}+j b / 2\right]^{2} \\
& \left.+\left[\left(k+\delta_{n+j}-\delta_{n}\right) c+w_{m+i, n+j, p+k}-w_{m n p}\right]^{2}\right\}^{1 / 2} .
\end{aligned}
$$

For numerical calculations we use the lattice parameters appropriate for crystalline polyethylene (PE). The PE crystal is formed by zigzag molecular chains $\left(\mathrm{CH}_{2}\right)_{\infty}$ with a longitudinal step $c=1.276 \AA$. The mass of one chain link is $M$ $=14 m_{p}$, where $m_{p}$ is the proton mass. The distance between 
adjacent chains is $a \simeq 4 \AA$, and period $b=a \sqrt{3}=6.928 \AA$. The longitudinal stiffness of PE can be estimated by the speed of long-wavelength acoustic phonons of trans-zigzag: $K_{\rho}=M\left(v_{0} / c\right)^{2}$. According to Refs. 22 and 23, the speed is $v_{0}=7819 \mathrm{~m} / \mathrm{s}$; hence $K_{\rho}=87 \mathrm{~N} / \mathrm{m}=523.9 \mathrm{~kJ} / \mathrm{mol} \AA^{2}$. The bending stiffness $K_{\theta}$ of a chain corresponds in our model to the stiffness of the torsion angle of trans-zigzag PE. According to Refs. 24 and $25, K_{\theta}=61.93 \mathrm{~kJ} / \mathrm{mol}$. Let us take $\varepsilon$ $=2 \mathrm{~kJ} / \mathrm{mol}$, and choose the parameter of the Morse potential [Eq. (4)] to be $\beta=5 \AA^{-1}$. Then from the set of equations (7) and (8), we obtain the values $\rho_{0}=4.072 \AA$ and $r_{0}$ $=1.298 \AA$.

\section{POINT DEFECTS AND TWISTED TOPOLOGICAL SOLITONS}

In fact, only a finite number of molecular chains participates in the formation of point defects of the crystalline structure given by Eqs. (9)-(14). Thus, for the formation of a vacancy (or an interstitial), it is sufficient to stretch (or to contract) only one chain of the crystal for one chain period. These defects can be described as topological solitons ${ }^{19}$ and, in the particular case of polyethylene crystal, their dynamics has been studied in the approximation of fixed neighbors ${ }^{23}$ and using molecular-dynamics simulations. ${ }^{26,27}$ These solitons have the width of some tens of a chain link, and an energy of about $40-50 \mathrm{~kJ} / \mathrm{mol}$.

Here we consider defects related to transverse (bending) deformations of the crystal chains, with one chain displaced to the place of another one or with the interlaced chains. Defects of this type are associated with the broken order of adjacent chains. A few chains must necessarily participate in their formation. Therefore, bending deformations of the chains are localized in space. Their existence is stipulated by a local violation of the topology of a polymer crystal, which is implemented through bending deformations of the chains. Therefore, in what follows we call these "twisted topological defects." Each of these defects is characterized by the number $N_{c} \geqslant 2$ of chains involved in the defect formation.

It should be noted that the "twisted topological defect" is not a new concept. Thus, for isotropic systems, such excitations have been studied in the context of a vortex lattice, ${ }^{28}$ where the two-chain defect is normally unstable while the three-chain defect is metastable. The instability here is due to the relatively low activation energy of breaking intersite bonds in the localization region of the defect. As a result, a reconnection of the twisted lines into a straight line occurs. In the polymer crystal studied in the present paper, a reconnection of this type is practically impossible because it requires a total breaking of the strong longitudinal valence bonds. Therefore, in a strongly anisotropic lattice, all the defects of twisting chains with strong bonds will be stable because the energy of longitudinal bonds exceeds that of transverse bonds by two orders. This circumstance allows us to consider the strong bonds as stable links. Thus, in the polymer crystal, only weak interchain (nonvalence) bonds can be broken rather than strong intrachain (valence) bonds.

The complexity of the model does not allow an analytical description of these defects. To find these numerically, one

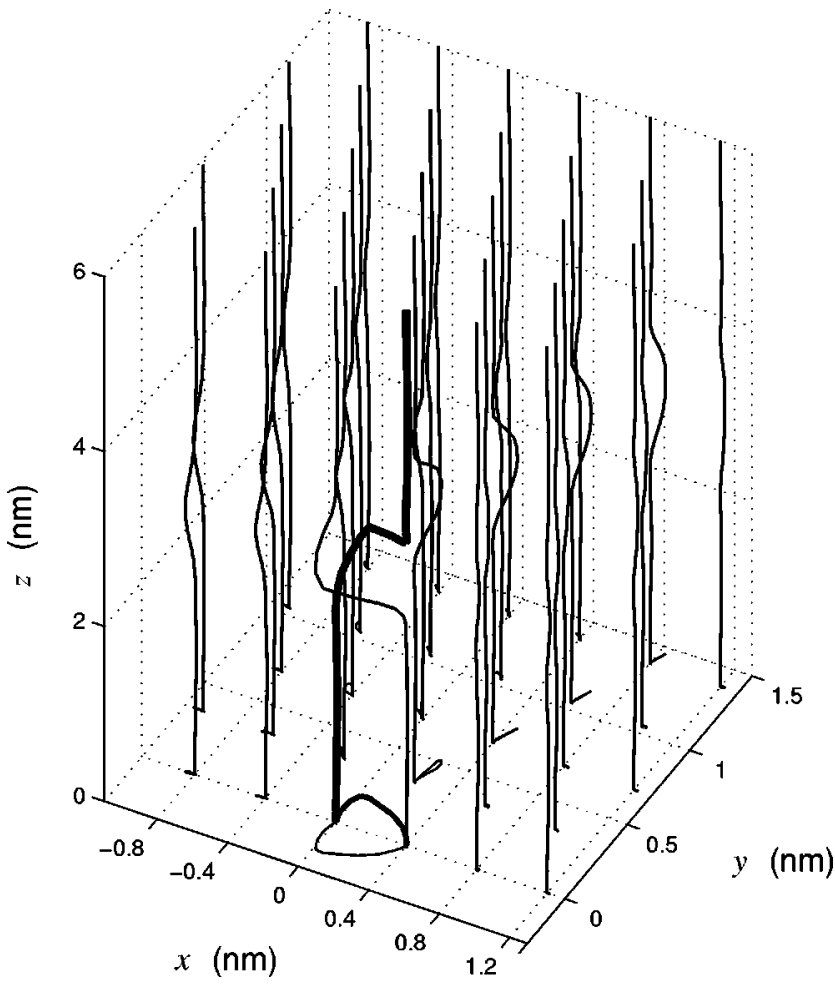

FIG. 2. Shape of the twisted topological defect formed by two adjacent chains $\left(N_{c}=2\right)$. For spatial visualization, projections of chains onto the $x y$ plane are included.

should restrict oneself to a consideration of a finite fragment of the crystal. Therefore we take a 3D rectangle

$$
\Lambda=\{1 \leqslant m \leqslant M, \quad 1 \leqslant n \leqslant N, \quad 1 \leqslant p \leqslant P\},
$$

and take into account only the interaction of particles at a distance $r \leqslant 7 a$.

To obtain a stationary state of a twisted topological defect, it is necessary to solve the minimization problem

$$
E \rightarrow \min _{(m, n, p) \in \Lambda},
$$

with periodic boundary conditions for transverse components $m$ and $n$, and the conditions of fixed ends for the longitudinal component $p$. Then problem (16) can be solved numerically by the conjugate-gradient method. The type of defect is determined by the assigned initial configuration of the crystal fragment [Eq. (15)].

First let us consider the twisted topological defect with $N_{c}=2$. In the region of localization of this defect, two crystal chains interchange their locations. To find a stationary profile of this defect, it is necessary to solve problem (16) with the initial configuration, with two interchanging chains given by

$$
\begin{gathered}
u_{M / 2, N / 2, p}=a\{1+\tanh [\mu(p-P / 2-1 / 2)]\} / 2, \\
v_{M / 2, N / 2, p}=b / 4 \cosh [\mu(p-P / 2-1 / 2)], \\
u_{M / 2, N / 2+1, p}=-u_{M / 2, N / 2, p}, \quad v_{M / 2, N / 2+1, p}=-v_{M / 2, N / 2, p}, \\
u_{m n p}=0, \quad v_{m n p}=0, \quad w_{m n p}=0 \quad \text { for other }(m, n, p) \in \Lambda .
\end{gathered}
$$



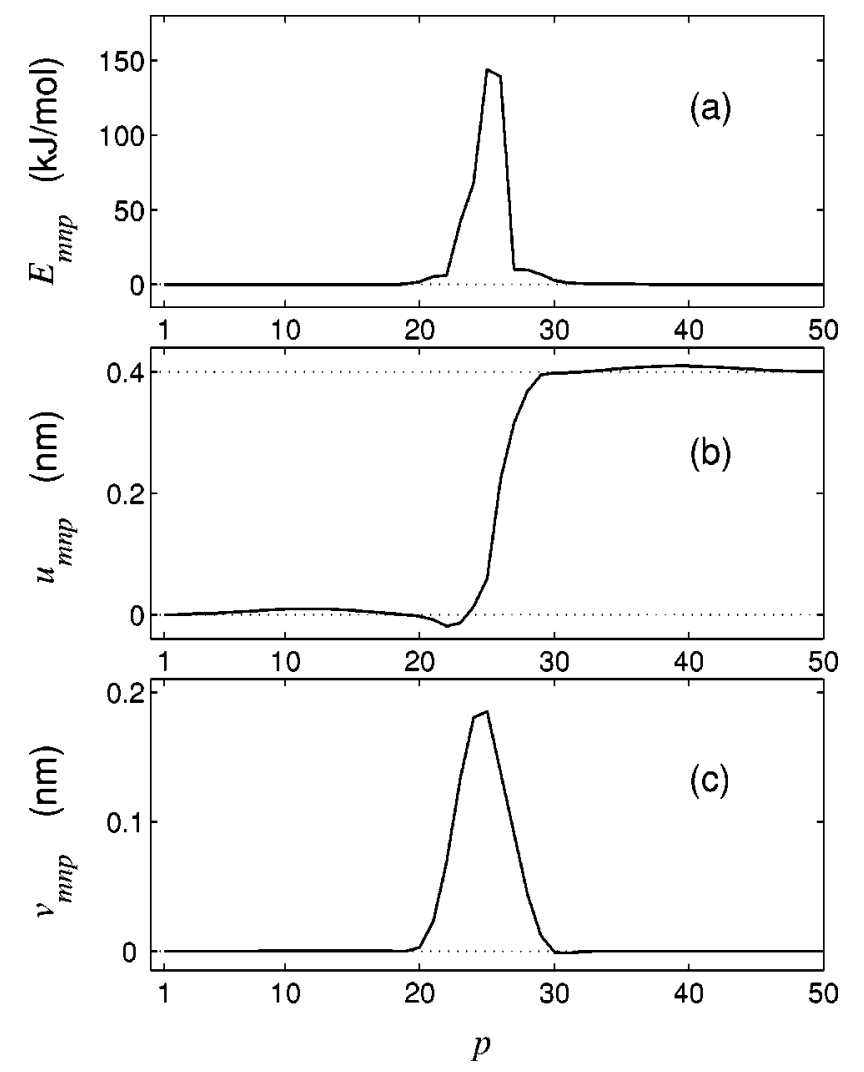

FIG. 3. Distribution of energy $E_{m n p}$ (a), and displacements $u_{m n p}$ (b) and $v_{m n p}$ (c) along one of the chains $(m=M / 2, n=N / 2)$ of a twisted topological defect with $\left(N_{c}=2\right)$.

In the numerical simulations we use $M=20, N=20$, and $P$ $=50$, and a dimensionless parameter characterizing the longitudinal width of the defect, $\mu=1 / 2$.

The form of the defect with $N_{c}=2$ is shown in Fig. 2. Two molecular chains of the crystal interchange their places without intersecting. This replacement causes a bending of adjacent chains. The projections of the chains onto the $x y$ plane are also shown in Fig. 2, to give visual representations of the amplitudes of the chain deformations. If we consider a two-dimensional strip with the edges formed by these two chains, then in the region of the defect localization it is overwound by $180^{\circ}$. Distributions of energy and displacements of sites, along a molecular chain participating in the formation of defects, are given in Fig. 3. The corresponding chain in Fig. 2 is shown by a bold line. In the $u$ component, the defect has the form of a step that describes the transition of a chain from one equilibrium position into the other equivalent adjacent equilibrium position. In the $v$ component, the displacement profile has the form of an isolated peak, describing the bypass of the other chain participating in the defect formation. As can be seen from Fig. 3, the defect is localized over ten chain bonds, where practically all the energy of the deformation is concentrated. The form of the defect does not depend smoothly on the number of chain bonds $p$. The defect is pinned, and it cannot move. The energy of the defect is $E_{2}=5220 \mathrm{~kJ} / \mathrm{mol}$. The pinning energy forms a barrier of 20 $\mathrm{kJ} / \mathrm{mol}$. In the localization region of the defect, the maximal energy per longitudinal bond does not exceed $150 \mathrm{~kJ} / \mathrm{mol}$,

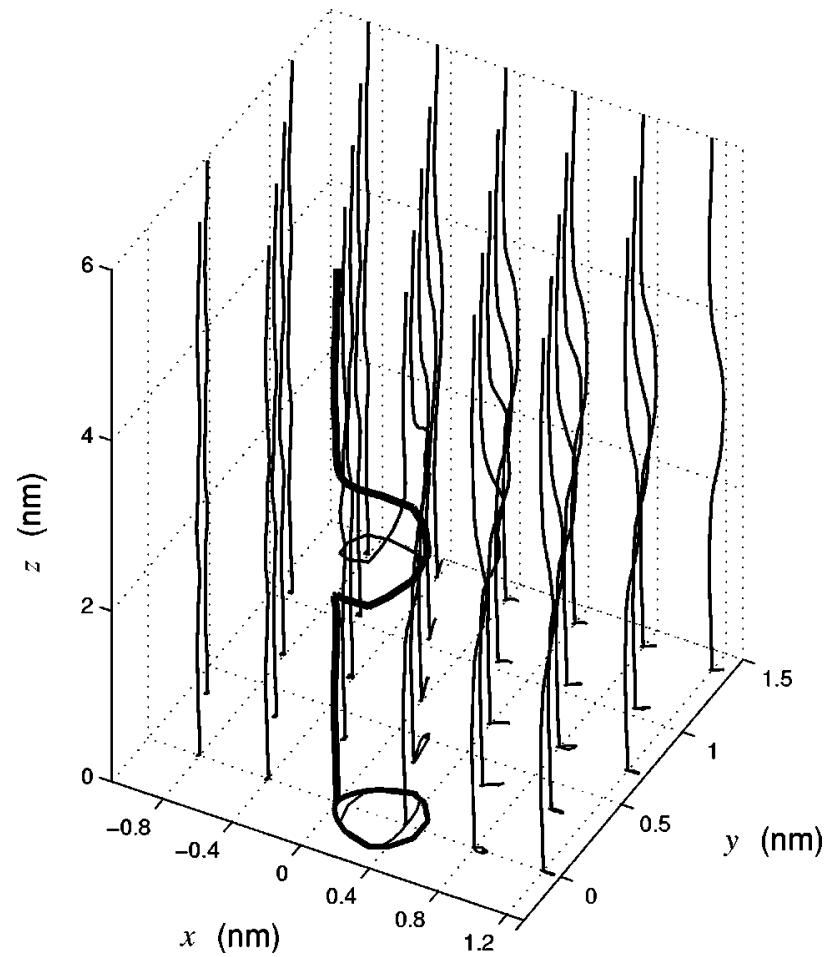

FIG. 4. Shape of a defect in the interlacing of two adjacent chains. For spatial visualization, the projections of chains onto the $x y$ plane are given.

and this is clearly not enough to break the chain [the energy of the valence bond $\mathrm{C}-\mathrm{C}$ is $529 \mathrm{~kJ} / \mathrm{mol}$ (Ref. 26)]. Therefore, here the chain reconnection is impossible resulting in the defect stability.

Let us consider the defect associated with the interlacing of two adjacent chains. To find a stationary profile of the defect, it is necessary to solve problem (16) with the initial configurations

$$
\begin{gathered}
\left.u_{M / 2, N / 2, p}=a / \cosh [\mu(p-P / 2-1 / 2)]\right\} / 2, \\
v_{M / 2, N / 2, p}=b / 4 \cosh [\mu(p-P / 2-1 / 2)], \\
u_{M / 2, N / 2+1, p}=-u_{M / 2, N / 2, p}, \quad v_{M / 2, N / 2+1, p}=-v_{M / 2, N / 2, p}, \\
u_{m n p}=0, \quad v_{m n p}=0, \quad w_{m n p}=0 \quad \text { for other }(m, n, p) \in \Lambda .
\end{gathered}
$$

The form of the defect is represented in Fig. 4. Here the two-dimensional strip with the edges formed by interlaced chains is overwinded by $360^{\circ}$. Since intrachain rigid intermolecular bonds cannot be torn, the molecular chains cannot pass through each other. Therefore, this defect also has a topological nature. The topological defect of this type can be considered as a bound state of the two twisted topological defects with $N_{c}=2$, each of them twisting the strip by $180^{\circ}$. Distributions of energy and displacements of the sites along one molecular chain participating in the defect formation are given in Fig. 5. The appropriate chain is shown in Fig. 4 by the bold line. The energy distribution along the chain has a distinctive two-hump profile. It is clearly seen from Fig. 4 that in the projection onto the $x y$ plane, this chain forms a 

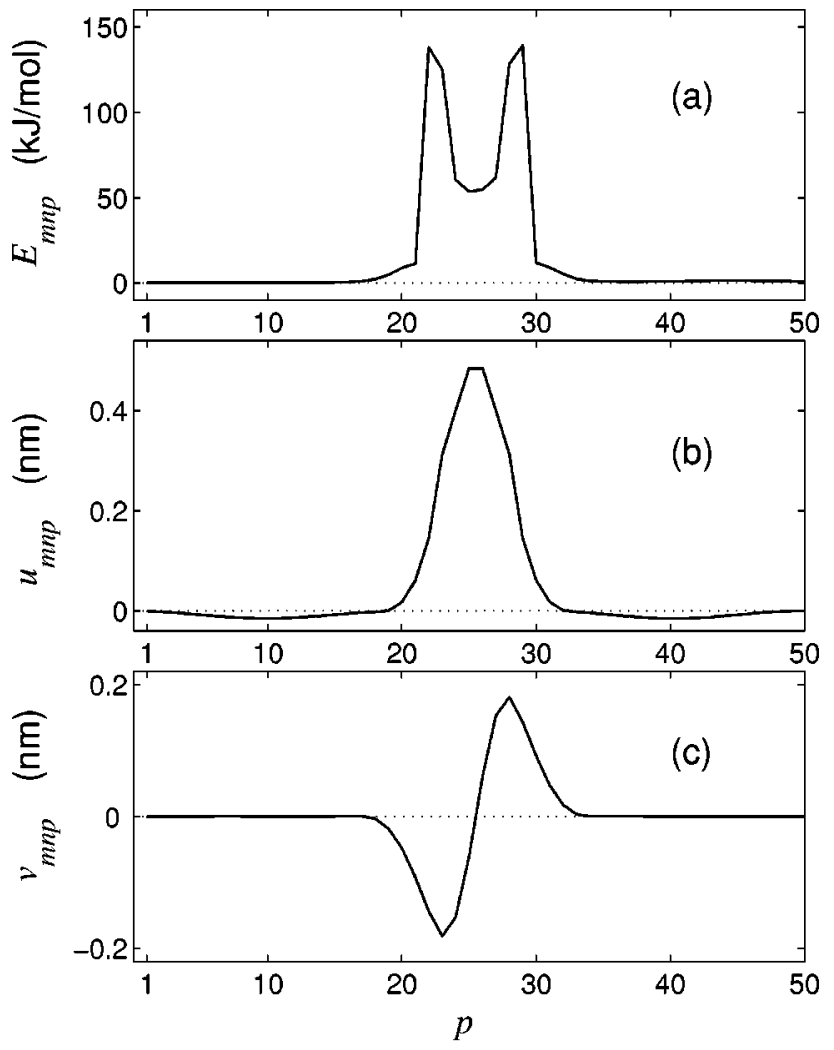

FIG. 5. Distribution of energy $E_{m n p}$ (a), and displacements $u_{m n p}$ (b) and $v_{m n p}$ (c) along one of the chains $(m=M / 2, n=N / 2)$ of an interlacing defect.

closed loop, surrounding the equilibrium position of the adjacent chain. The energy of an interlacing defect $\left(E_{1}\right.$ $=8977 \mathrm{~kJ} / \mathrm{mol}$ ) is less than the energy of two twisted topological defects $\left(2 E_{2}=10440 \mathrm{~kJ} / \mathrm{mol}\right)$. The binding energy of these two defects can be estimated as $\Delta E=2 E_{2}-E_{1}$ $=473 \mathrm{~kJ} / \mathrm{mol}$.

The twisted topological defect can be formed by more than two chains. The only important point is that they must sequentially occupy each others positions (their projection onto the $x y$ plane must form a continuous closed loop passing through the sites of a 2D hexagonal lattice, corresponding to equilibria of the chains). The dependence of the defect energy $E$ on the number of chains $N_{c}$ is represented in Table I. The defect with the least energy $E=3790 \mathrm{~kJ} / \mathrm{mol}$ has $N_{c}$ $=6$, for which the molecular chains form an exact hexagon in the projection onto the $x y$ plane (Fig. 6). The distribution of the energy and the displacements along one chain of this defect are presented in Fig. 7 (this chain is shown with bold line in Fig. 6). Here all the displacement components have smooth profiles typical of a soliton.

TABLE I. Dependence of the energy $E$ of a twisted topological defect on the number of chains $N_{c}$ forming a defect.

\begin{tabular}{lccccc}
\hline \hline$N_{c}$ & 2 & 3 & 4 & 5 & 6 \\
\hline$E(\mathrm{~kJ} / \mathrm{mol})$ & 5220 & 4264 & 4512 & 5094 & 3790 \\
\hline \hline
\end{tabular}

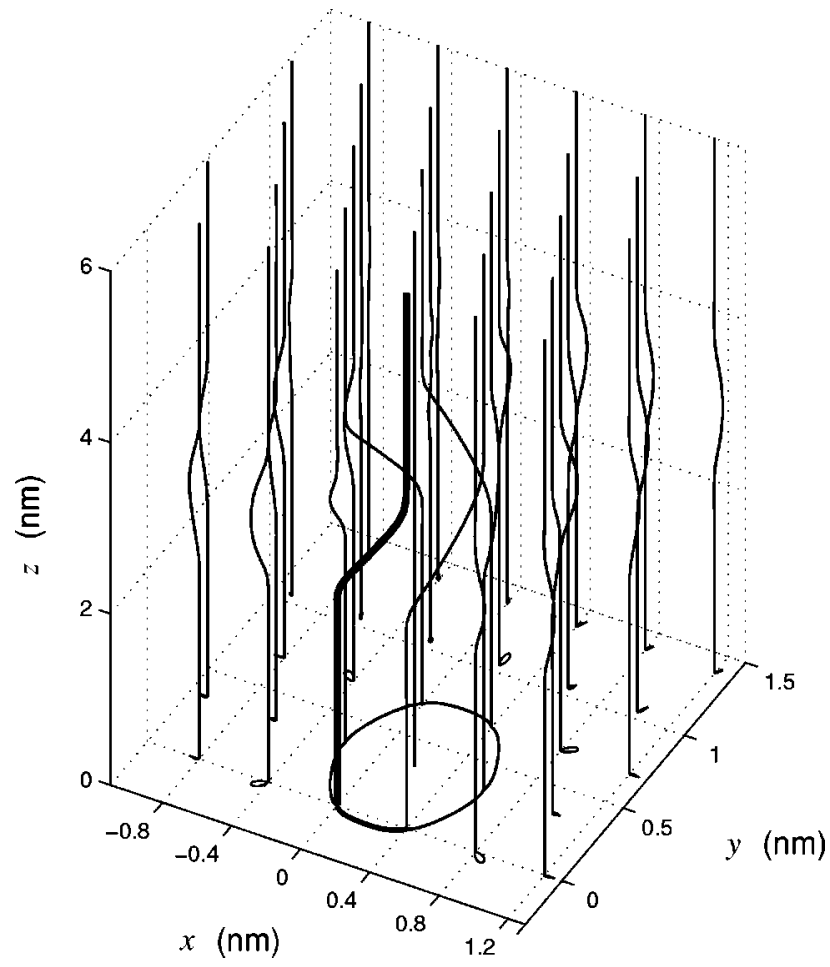

FIG. 6. Shape of a twisted topological defect formed by six chains $\left(N_{c}=6\right)$. For spatial visualization, the projections of chains onto the $x y$ plane are given.

\section{DEFECT DYNAMICS}

The twisted topological defect with $N_{c}=2$ has no smooth profile, as demonstrated in Fig. 3. It is pinned, and cannot propagate as a solitary wave. However, at $N_{c}=6$, the defect has a broad and smooth profile, and therefore it can move. For convenience of numerical simulations of the defect motion, we introduce the dimensionless time $\tau=t / t_{0}$, where $t_{0}$ $=c / v_{0}=\sqrt{M / K_{\rho}}$ is the time required for the sound to pass one chain bond. To obtain the profile of a moving soliton, we use the method of minimization of a discrete Lagrangian. ${ }^{18}$ The soliton profile $\left\{u_{m n p}, v_{m n p}, w_{m n p}\right\}$ can be found as a minimum of the discrete Lagrangian

$$
\begin{aligned}
L= & E-\frac{1}{2} s^{2} K_{\rho} \sum_{(m, n, p) \in Z^{3}}\left[\left(u_{m n, p+1}-u_{m n p}\right)^{2}\right. \\
& \left.+\left(v_{m n, p+1}-v_{m n p}\right)^{2}+\left(w_{m n, p+1}-w_{m n p}\right)^{2}\right],
\end{aligned}
$$

where the potential energy $E$ of the system is defined by expression (10), and the dimensionless speed of a soliton is $s=v / v_{0}$.

Upon using the dimensionless time $\tau$, the set of equations of motion takes the forms

$$
\begin{gathered}
K_{\rho} u_{m n p}^{\prime \prime}=-\partial H / \partial u_{m n p}, \\
K_{\rho} v_{m n p}^{\prime \prime}=-\partial H / \partial v_{m n p}, \\
K_{\rho} w_{m n p}^{\prime \prime}=-\partial H / \partial w_{m n p}, \\
(m, n, p) \in \Lambda,
\end{gathered}
$$



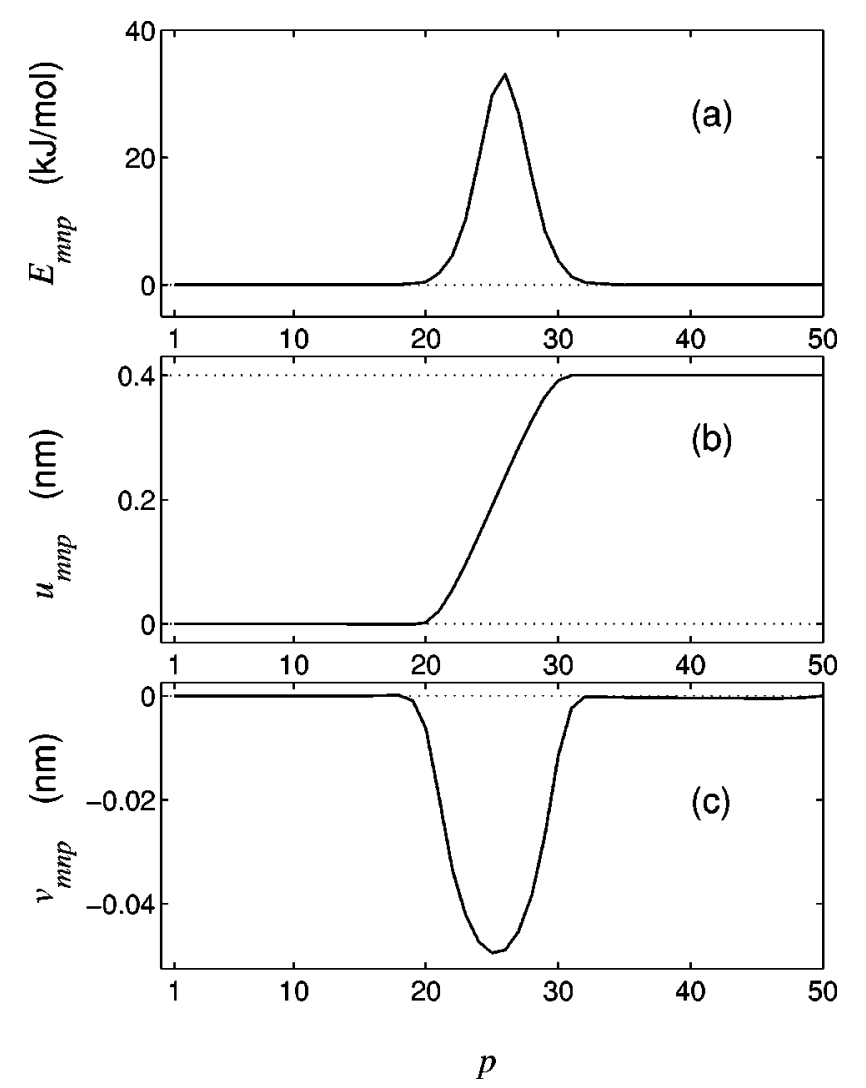

FIG. 7. Distribution of energy $E_{m n p}$ (a), and displacements $u_{m n p}$ (b) and $v_{m n p}$ (c) along one of the chains $(m=M / 2, n=N / 2)$ of a twisted topological defect $\left(N_{c}=6\right)$.

where the Hamiltonian $H$ of the system is given by Eq. (9). Then we solve the problem

$$
L \rightarrow \min _{(m, n, p) \in \Lambda}
$$

at $s=0.5$, using an initial configuration corresponding to the twisted topological soliton with $N_{c}=6$. The solution $\left\{u_{m n p}, v_{m n p}, w_{m n p}\right\}$ of this problem can be used as an initial approximation for the system of equations of motion (20):

$$
\begin{gathered}
u_{m n p}^{\prime}(0)=-s\left(u_{m n, p+1}-u_{m n p}\right), \\
v_{m n p}^{\prime}(0)=-s\left(v_{m n, p+1}-v_{m n p}\right), \\
w_{m n p}^{\prime}(0)=-s\left(w_{m n, p+1}-w_{m n p}\right), \\
u_{m n p}(0)=u_{m n p}, \quad v_{m n p}(0)=v_{m n p}, \\
w_{m n p}(0)=w_{m n p}, \quad(m, n, p) \in \Lambda .
\end{gathered}
$$

The defect dynamics is presented in Fig. 8. It is clearly seen that the defect moves along molecular chains of the crystal as a solitary wave with a stationary speed $s=0.276$, retaining its form. Thus, in the polymer crystal, in addition to well-defined longitudinal topological solitons, transverse topological solitons do exist.

Topological defects with $N_{c} \geqslant 6$ have relatively low energy densities in their localization region, as well as high
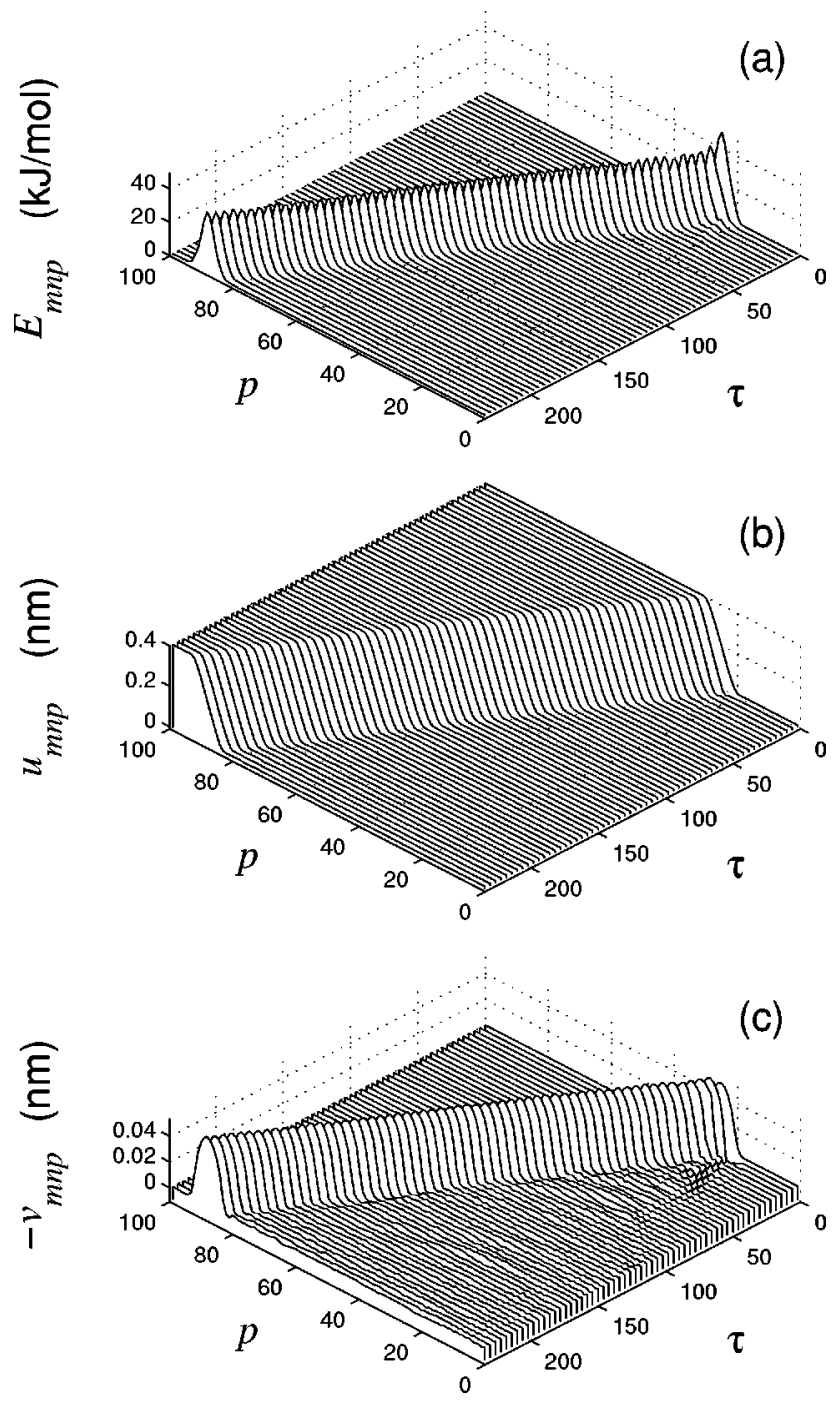

FIG. 8. Dynamics of a twisted topological defect with $N_{c}=6$. Distribution of energy $E_{m n p}$ (a), and displacements $u_{m n p}$ (b) and $v_{m n p}$ (c) along the chain $m=M / 2$ and $n=N / 2$ of the defect.

mobilities. Therefore, they can be thermally activated as pairs of opposite polarity. These defects can be separated each from each other by large distances caused by lattice dislocation loops, which are important for melting. ${ }^{29}$ Thus twisted topological solitons should play an important role in premelting mechanisms for strongly anisotropic polymer crystals.

\section{DISLOCATIONS IN POLYMER CRYSTAL}

The model used in the previous sections also allows a consideration of dislocations in a polymer crystal. The edge dislocation associated with longitudinal deformations of molecular chains of the crystal was already investigated, ${ }^{19}$ and therefore here we will focus only on a study of the dislocations associated with bending deformations of the molecular chains.

To find the stationary profile of a dislocation, we choose the finite rectangular fragment of the crystal [Eq. (15)], with periodic boundary conditions in all three indices $m, n$, and $p$. 


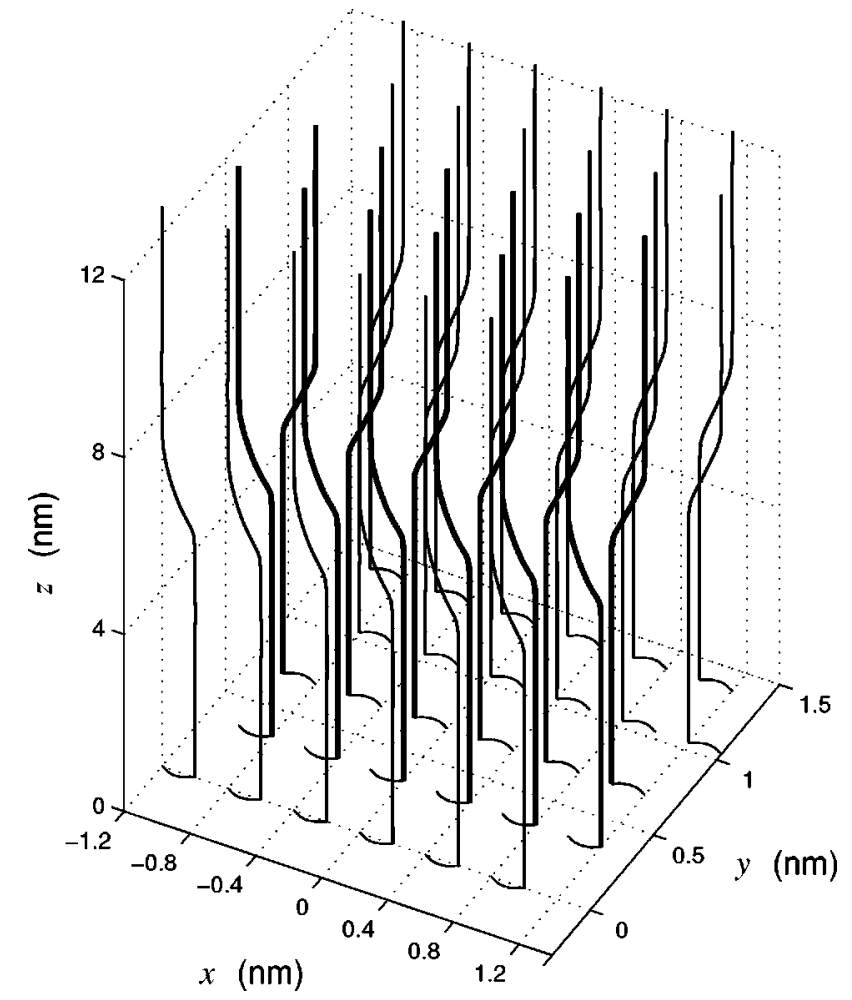

FIG. 9. Shape of molecular chain deformations within the core of the screw dislocation with the Burgers vector $\mathbf{b}=(a, 0,0)$. Chains nearest to the slip plane are shown by bold lines.

The periodic boundary conditions do not allow us to obtain one isolated dislocation, but we can find a dislocation quadrupole: four dislocations with the sum of the Burgers vectors equal to 0 . At sufficiently large distances between the dislocation centers, they can be considered as separate dislocations.

Let us first consider the screw dislocation with the Burgers vector $\mathbf{b}=(a, 0,0)$. In a crystal with such a dislocation, all the chains with an identical index $m$ form identical deformations (see Fig. 9). The dislocation line is parallel to the $x$ axis. Therefore, one can omit the index $m$ everywhere, having put

$$
\begin{gathered}
u_{m n p} \equiv u_{1 n p}=u_{n p}, \\
v_{m n p} \equiv v_{1 n p}=v_{n p}, \\
w_{m n p} \equiv w_{1 n p}=w_{n p} .
\end{gathered}
$$

The stationary profile of a dislocation quadrupole was found to be a solution of the minimization problem [Eq. (16)] with $M=1, N=200$, and $P=200$, and with the initial configurations

$$
u_{n, p}=a \tanh [\mu(p-P / 4-1 / 2)] / 2, \quad u_{n, p+P / 2}=-u_{n, p},
$$

for

$$
N / 4<n \leqslant 3 N / 4, \quad 1 \leqslant p \leqslant P / 2,
$$
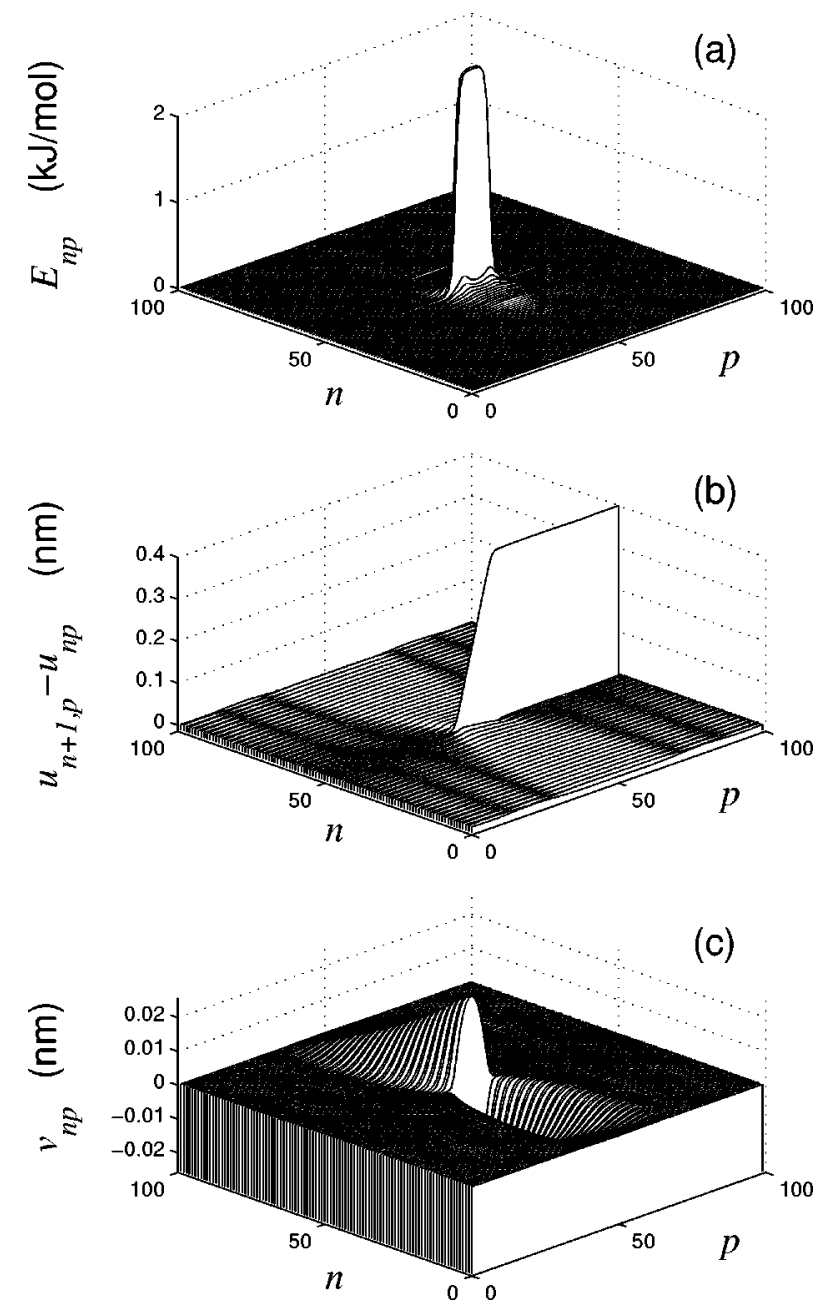

FIG. 10. Distribution of energy $E_{n p}$ (a), relative displacements $u_{n+1, p}-u_{n p}$ (b), and transverse displacements $v_{n p}$ (c) in the lattice with the screw dislocation. The Burgers vector is $\mathbf{b}=(a, 0,0)$.

$$
u_{n, p}=-a \tanh [\mu(p-P / 4-1 / 2)] / 2, \quad u_{n, p+P / 2}=-u_{n, p},
$$

for

$$
1 \leqslant n \leqslant N / 4, \quad 3 N / 4+1 \leqslant n \leqslant N, \quad 1 \leqslant p \leqslant P / 2,
$$

and

$$
v_{n, p}=0, \quad w_{n p}=0, \quad \text { for } 1 \leqslant n \leqslant N, \quad 1 \leqslant p \leqslant P .
$$

In the rectangular fragment $1 \leqslant n \leqslant N / 2,1 \leqslant p \leqslant / 2 P$, we obtain one screw dislocation.

Deformations of molecular chains in the dislocation core are shown in Fig. 9. Distributions of the energy and the displacements in the rectangular region $1 \leqslant n \leqslant 100,1 \leqslant p \leqslant 100$ are presented in Fig. 10. In this rectangular region of a crystal of width $a$, the dislocation has the energy $E$ $=76.3 \mathrm{~kJ} / \mathrm{mol}$ (all dislocations in a quadrupole have identical energy). It is evident from Fig. 10(a) that most of the dislocation energy is concentrated in the two layers where the relative displacement of chains by one period along the $x$ axis takes place. The chains in these layers are shown by bold lines in Fig. 9. Figure 10(b) shows that in other layers the chains are practically not displaced with regard to each 


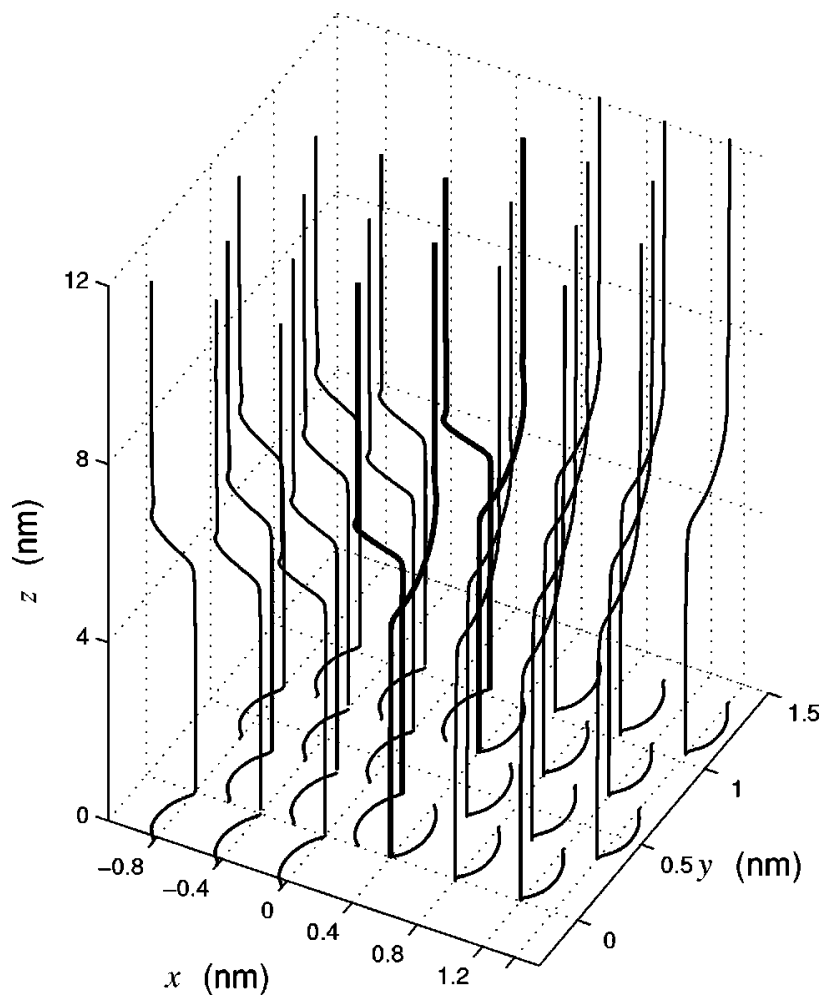

FIG. 11. Shape of molecular chain deformations within the core of the screw dislocation with the Burgers vector $\mathbf{b}=(0, b, 0)$. Chains nearest to the slip plane are shown by bold lines.

other. The width of the dislocation core is about 15 periods of a chain. The interchain interaction leads to slight displacements along the $y$ axis in the dislocation core region, as illustrated by Fig. 10(c). As a result, the projections of chains onto the $x y$ plane have characteristic arc forms, as seen in Fig. 9.

Upon formation of the screw dislocation with the Burgers vector $\mathbf{b}=(0, b, 0)$, all chains with odd and even indices $n$ are equally deformed (Fig. 11). Here the dislocation line is parallel to the $y$ axis. Therefore, for finding a dislocation of this type, it is sufficient to take the rectangular fragment of the crystal $\Lambda$ with $N=2$. Let us take $M=100$ and $P=200$, and solve the minimization problem [Eq. (16)] with the initial configuration of the fragment $\Lambda$, appropriate to a dislocation quadrupole.

The shape of chain deformations in the core of one dislocation is shown in Fig. 11. Distributions of the energy and the displacements in the rectangular region, $1 \leqslant m \leqslant 50$ and $1 \leqslant p \leqslant 100$, are presented in Fig. 12. As can be seen from Fig. 12(a), the energy is no longer localized near the slip plane (the chains in these layers are shown with bold lines in Fig. 11). The width of the dislocation core is about 20 [Fig. $12(\mathrm{c})]$ periods of the chain. Along the $x$ axis, the distances between chains in the dislocation core are much larger [Fig. 12(b)], and consequently the projections of chains onto the $x y$ plane have more pronounced arc shapes (Fig. 11). In the rectangular region of the crystal, $1 \leqslant m \leqslant 50,1 \leqslant n \leqslant 2$, and $1 \leqslant p \leqslant 100$, the dislocation with the Burgers vector $\mathbf{b}$ $=(0, b, 0)$ has a considerably higher energy $E$ $=775.3 \mathrm{~kJ} / \mathrm{mol}$. This stipulates the possibility for such a dis-
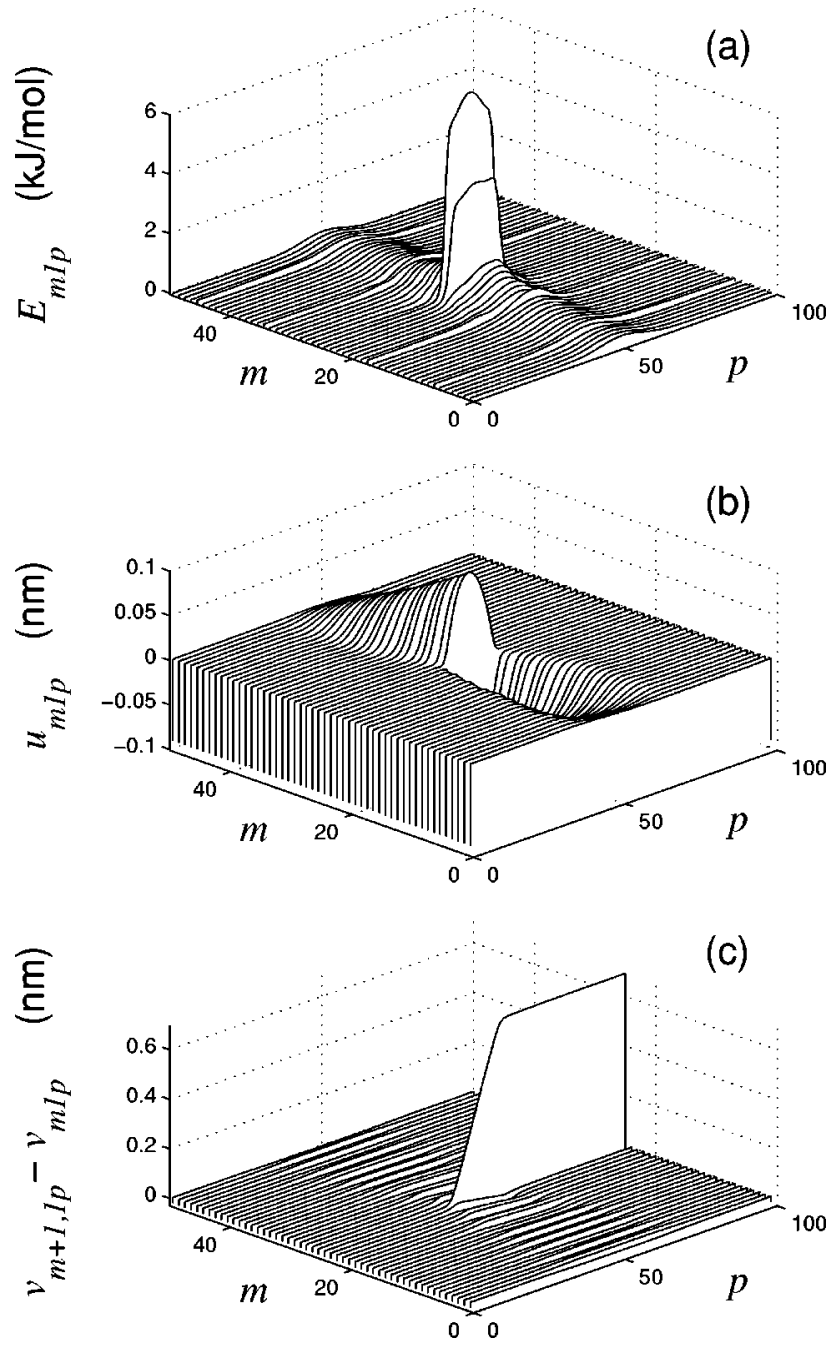

FIG. 12. Distribution of energy $E_{m 1 p}$ (a), transverse displacements $u_{m 1 p}(\mathrm{~b})$, and relative displacements $v_{m+1,1, p}-v_{m 1 p}$ (c) in the lattice with the screw dislocation. The Burgers vector is $\mathbf{b}$ $=(0, b, 0)$.

location to be unstable concerning the dissociation into two mixed dislocations with the Burgers vectors of the type $(a, 0,0)$.

Let us now consider an edge dislocation with the Burgers vector $\mathbf{b}=(0,0, c)$ and with a line parallel to the $x$ axis. Again, we exploit symmetry condition (23), and solve mini mization problem (16) with $M=1, N=100$, and $P=400$ with the initial configuration

$$
\begin{gathered}
w_{n, p}=c\{1+\tanh [\mu(p-P / 4-1 / 2)]\} / 2, \\
w_{n, P / 2+p}=w_{n, P / 2+1-p},
\end{gathered}
$$

for

$$
\begin{gathered}
N / 4<n \leqslant 3 N / 4, \quad 1 \leqslant p \leqslant P / 2, \\
w_{n, p}=0,
\end{gathered}
$$

for

$$
1 \leqslant n \leqslant N / 4, \quad 3 N / 4+1 \leqslant n \leqslant N, \quad 1 \leqslant p \leqslant P / 2,
$$



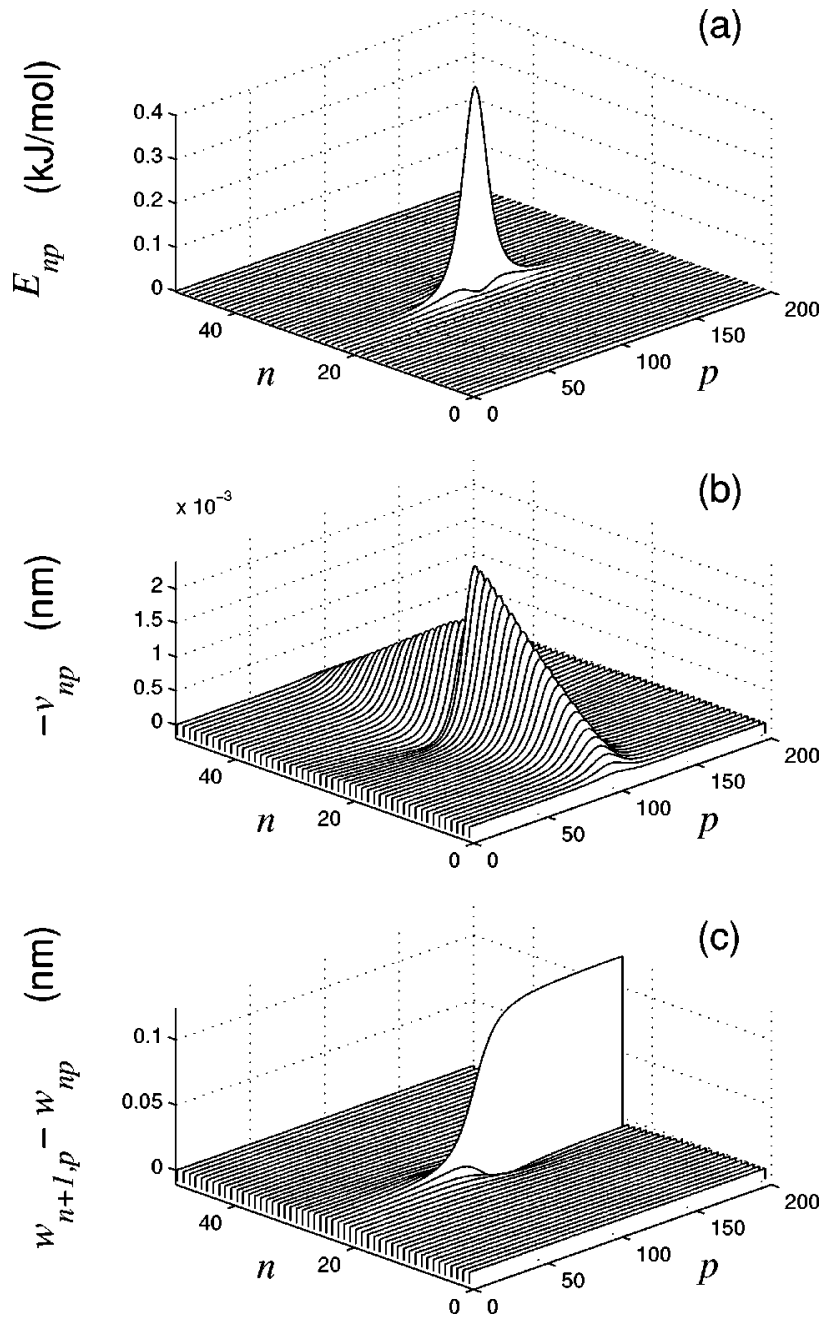

FIG. 13. Distribution of energy $E_{n p}$ (a), displacements $v_{n p}$ (b), and relative displacements $w_{n+1, p}-w_{n p}$ (c) in the lattice with the edge dislocation. The Burgers vector is $\mathbf{b}=(0,0, c)$, and the dislocation line is parallel to the $x$ axis.

$$
u_{n, p}=0, \quad v_{n, p}=0, \quad \text { for } 1 \leqslant n \leqslant N, \quad 1 \leqslant p \leqslant P .
$$

For the rectangular fragment $1 \leqslant n \leqslant N / 2,1 \leqslant p \leqslant P / 2$, we obtain one edge dislocation.
The shape of the solution is shown in Fig. 13. For the rectangular region of the crystal with $m=1,1 \leqslant n \leqslant 50$, and $1 \leqslant p \leqslant 100$, the edge dislocation has the energy $E$ $=22.6 \mathrm{~kJ} / \mathrm{mol}$. The energy distribution in a crystal is represented in Fig. 13(a). The main energy of a dislocation is concentrated in the layer with $n=N / 4$ that contains the dislocation core. The width of the dislocation core appears to be more than 50 chain bonds [Fig. 13(c)]. The shape of displacements in the transverse component $v$ is shown in Fig. 13(b).

The formation of edge dislocations with the Burgers vector parallel to the $x y$ plane takes place without deformations of the molecular chains. The chains are displaced as a whole, and therefore, in the projection onto the $x y$ plane, these dislocations correspond to dislocations of a $2 \mathrm{D}$ hexagonal crystal.

\section{CONCLUSIONS}

We have shown in the present paper that the polymer crystal can contain local topological defects possessing soliton dynamics. Along with the well-known longitudinal topological solitons, there exist twisted topological solitons, exhibiting local violations of crystal topology associated with bending deformations of molecular chains of the crystal (interlacing of chains, substitution of chains, etc.). Bending deformations of the chains are also responsible for the existence of screw dislocations, with the Burgers vector perpendicular to the direction of molecular chains of the crystal. It is shown that screw dislocations have higher energies than edge dislocations, the existence of which is associated with longitudinal deformations of molecular chains in the polymer crystal.

\section{ACKNOWLEDGMENTS}

This work was partially carried out with financial support from the European Union under the INTAS Grant No. 970368. Three of us (A.V.S., J.M.K., and A.V.Z.) would also like to express gratitude to the Section of Mathematical Physics at IMM of the Technical University of Denmark for financial support and hospitality.
${ }^{1}$ J. Frenkel and T. Kontorova, Phys. Z. Sowjetunion 13, 1 (1938).

${ }^{2}$ F.C. Frank and J.H. van der Merwe, Proc. R. Soc. London, Ser. A 198, 205 (1949).

${ }^{3}$ For a review, see, e.g., A. M. Kosevich, Physical Mechanics of Realistic Crystals (Naukova Dumka, Kyiv, 1981).

${ }^{4}$ See, e.g., A. H. Cottrell, Theory of Crystal Dislocation (Blackie and Son, London, 1964).

${ }^{5}$ J. P. Hirth and J. Lothe, Theory of Dislocations (Wiley, New York, 1968).

${ }^{6} \mathrm{~S}$. Aubry, in Solitons in Condensed Matter Physics, edited by A. R. Bishop and T. Schneider (Springer, Berlin, 1978), p. 264.

${ }^{7}$ A.R. Bishop, J.A. Krumhansl, and S.E. Trullinger, Physica D 1, 1 (1980).

${ }^{8}$ For a review, see, e.g., L.V. Yakushevich, Physica D 79, 77
(1994).

${ }^{9}$ W.T. Sanders, J. Appl. Phys. 36, 2822 (1965).

${ }^{10}$ J. Pouget, S. Aubry, A.R. Bishop, and P.S. Lomdahl, Phys. Rev. B 39, 9500 (1989).

${ }^{11}$ J.M. Tamga, M. Remoissenet, and J. Pouget, Phys. Rev. Lett. 75, 357 (1995).

${ }^{12}$ P.S. Lomdahl and D.J. Srolovitz, Phys. Rev. Lett. 57, 2702 (1986).

${ }^{13}$ D.J. Srolovitz and P.S. Lomdahl, Physica D 23, 402 (1986).

${ }^{14}$ Y. Zolotaryuk, A.V. Savin, and P.L. Christiansen, Phys. Rev. B 57, 14213 (1998).

${ }^{15}$ O.M. Braun and Y.S. Kivshar, Phys. Rev. B 44, 7694 (1991).

${ }^{16}$ O.M. Braun, O.A. Chubykalo, Y.S. Kivshar, and L. Vázquez, Phys. Rev. B 48, 3734 (1993). 
${ }^{17}$ P.L. Christiansen, A.V. Savin, and A.V. Zolotaryuk, Phys. Rev. B 54, 12892 (1996).

${ }^{18}$ P.L. Christiansen, A.V. Savin, and A.V. Zolotaryuk, J. Comput. Phys. 134, 108 (1997).

${ }^{19}$ P.L. Christiansen, A.V. Savin, and A.V. Zolotaryuk, Phys. Rev. B 57, 13564 (1998).

${ }^{20}$ J. Pouget, Phys. Rev. B 43, 3575 (1991); 43, 3582 (1991).

${ }^{21}$ J. Pouget, Phys. Rev. B 46, 10554 (1992).

${ }^{22}$ L.I. Manevitch and A.V. Savin, Phys. Rev. E 55, 4713 (1997).

${ }^{23}$ A.V. Savin and L.I. Manevitch, Phys. Rev. B 58, 11386 (1998).
${ }^{24}$ D.W. Noid, B.G. Sumpter, and B. Wunderlich, Macromolecules 24, 4148 (1991).

${ }^{25}$ B.G. Sumpter, D.W. Noid, G.L. Liang, and B. Wunderlich, Adv. Polym. Sci. 116, 29 (1994).

${ }^{26}$ F. Zhang, Phys. Rev. E 56, 6077 (1997).

${ }^{27}$ F. Zhang, Phys. Rev. B 59, 792 (1999).

${ }^{28}$ A. Schönenberger, V. Geshkenbein, and G. Blatter, Phys. Rev. Lett. 75, 1380 (1995).

${ }^{29}$ M.C. Marchetti and D.R. Nelson, Phys. Rev. B 90, 1910 (1990). 